\title{
Inertial effects in anomalous dielectric relaxation of symmetrical top molecules
}

\author{
Sergey V. Titov \\ Institute of Radio Engineering and Electronics of the Russian Academy of Sciences, Vvedenskii Square 1, Fryazino, Moscow Region, \\ 141190, Russian Federation \\ Yuri P. Kalmykov \\ MEPS, Groupe de Physique Moléculaire, Université de Perpignan, 52, Avenue Paul Alduy, 66860 Perpignan Cedex, France \\ William T. Coffey \\ Department of Electronic and Electrical Engineering, Trinity College, Dublin 2, Ireland
}

(Received 23 October 2003; published 31 March 2004)

\begin{abstract}
The linear dielectric response of an assembly of noninteracting symmetrical top molecules (each of which is free to rotate in space) is evaluated in the context of fractional dynamics. The infinite hierarchy of differentialrecurrence relations for the relaxation functions appropriate to the dielectric response is derived by using the underlying inertial fractional Klein-Kramers equation. On solving this hierarchy in terms of matrix continued fractions (as in the normal rotational diffusion), the complex dynamic susceptibility is obtained and is calculated for typical values of the model parameters. For the limiting case of spherical top molecules, the solution is obtained in terms of an ordinary continued fraction. It is shown that the model can reproduce nonexponential anomalous dielectric relaxation behavior at low frequencies ( $\omega \tau \leqslant 1$, where $\tau$ is the Debye relaxation time) and the inclusion of inertial effects ensures that optical transparency is regained at very high frequencies (in the far infrared region) so that Gordon's sum rule for integral dipolar absorption is satisfied.
\end{abstract}

DOI: 10.1103/PhysRevE.69.031114

\section{INTRODUCTION}

Dielectric relaxation of a variety of materials (such as amorphous polymers, glass forming liquids, etc.) often exhibits in practice nonexponential behavior with a slowly decaying long time tail. Such a process is commonly characterized by a complex susceptibility $\chi(\omega)$ which exhibits ColeCole behavior, viz.,

$$
\frac{\chi(\omega)}{\chi^{\prime}(0)}=\frac{1}{1+(i \omega \tau)^{\sigma}} \quad(0<\sigma<1)
$$

[for $\sigma=1$, Eq. (1) reduces to the well-known Debye equation]. Anomalous relaxation has its origins in anomalous diffusion. A physical explanation of anomalous diffusion described by Eq. (1) may be given in terms of the continuous time random walk (CTRW). The concept of a CTRW was introduced by Montroll and Weiss in 1965 [1,2] as a way to render time continuous in a random walk without an appeal to the diffusion or continuum limit. In the context of dielectric relaxation, the CTRW allows one to derive a fractional rotational diffusion equation in configuration space which yields Eq. (1) (detailed discussion of this subject is given in Ref. [3]). However, in such an approach inertial effects are ignored.

The omission of inertial effects in the relaxation process gives rise, in the context of dielectric relaxation, to the phenomenon of infinite dielectric absorption at high frequencies. In order to give a physically meaningful description of the high-frequency behavior (e.g., to avoid a diverging absorption coefficient), the inertial effects must also be included in anomalous relaxation just as in normal relaxation. In the context of Brownian motion, the inertial effects may be treated by either averaging the inertial Langevin equation over its
PACS number(s): 05.40.Jc, 77.22.-d, 05.45.Df

realizations or by constructing the Klein-Kramers equation for the evolution of the probability density function in phase space. We remark that models of the rotational Brownian motion are frequently used in studying orientational relaxation in liquids by various probe techniques such as dielectric relaxation, the dynamic Kerr effect, far-infrared absorption, Raman scattering, etc. [4-6]. The various generalizations of the Klein-Kramers equation to fractional diffusion which have been proposed have been described by Metzler and Klafter [7,8] and Barkai and Silbey [9]. By using the rotational counterpart of the fractional Klein-Kramers equation (FKKE) of Barkai and Silbey (their FKKE was suggested for one-dimensional translational Brownian motion), Coffey et al. $[10,11]$ have shown (by generalizing the approach given by Gross [12] and Sack $[13,14]$ for normal rotational diffusion) how inertial effects may be included in the fractional dynamics of noninteracting planar rotators and rotators in space (linear molecules) to yield a physically acceptable description of the far-infrared absorption in dipolar fluids. Moreover, Coffey et al. [10,11] have proposed a method of solution of the inertial FKKE. The key step in applying the method is first to convert by appropriate transformation the FKKE into an equation for the quantity the statistical average of which is desired. The resulting differential-recurrence relation may then be solved by continued fraction methods to yield the complex susceptibility.

Although the models considered in Refs. [10,11] reproduce the principal features of dielectric relaxation of an ensemble of dipolar molecules, these models may only be used in the limiting case of linear molecules. Here, the approach for rigid rotators proposed in Refs. $[10,11]$ is extended to the orientational relaxation of an assembly of dipolar nonpolarizable symmetrical top molecules undergoing fractional diffusion in space (treated originally by Morita [15] and Coffey 
et al. [16] for normal diffusion). Here we demonstrate how this generalized model can reproduce the nonexponential (Cole-Cole type) anomalous dielectric relaxation behavior and how the unphysical high frequency behavior of the absorption coefficient due to the neglect of inertia may be removed in fractional relaxation. The after effect solution for the dynamic Kerr effect [6] may be treated in analogous fashion and is also presented.

\section{ANOMALOUS ROTATIONAL DIFFUSION OF A SYMMETRICAL TOP MOLECULE IN SPACE}

The rotational Brownian motion of a symmetric top molecule in the molecular coordinate system oxyz rigidly connected to the top is characterized by the angular velocity $\boldsymbol{\omega}$ and the angular momentum $\mathbf{M}$ defined as [4]

$$
\boldsymbol{\omega}=\left(\omega_{x}, \omega_{y}, \omega_{z}\right)=(\dot{\vartheta}, \dot{\varphi} \sin \vartheta, \dot{\psi}+\dot{\varphi} \cos \vartheta)
$$

and

$$
\mathbf{M}=\left(I \omega_{x}, I \omega_{y}, I_{z} \omega_{z}\right),
$$

where $I$ and $I_{z}$ are the moments of inertia about the axis of symmetry and about an axis perpendicular to that axis, respectively, $\vartheta, \varphi$, and $\psi$ are the Euler angles $(\vartheta$ is the angle between the axis of symmetry of the molecule and the $Z$ axis of the laboratory coordinate system, $\varphi$ is the azimuthal angle, and $\psi$ is the angle characterizing rotation about the axis of symmetry).

In order to describe the fractional Brownian rotational motion, we use the FKKE for the evolution of the probability density function $W$ in configuration-angular velocity space for symmetrical top molecules in the same form as in Ref. [11] for linear molecules. For symmetric top molecules, the FKKE becomes (in the absence of external fields)

$$
\begin{aligned}
\frac{\partial W}{\partial t}+ & \omega_{x} \frac{\partial W}{\partial \vartheta}+\left(\omega_{y} \cot \vartheta-\frac{I_{z}}{I} \omega_{z}\right)\left(\omega_{y} \frac{\partial W}{\partial \omega_{x}}-\omega_{x} \frac{\partial W}{\partial \omega_{y}}\right) \\
= & \tau^{1-\alpha}{ }_{0} D_{t}^{1-\alpha}\left[\beta \frac{\partial}{\partial \omega_{x}}\left(\omega_{x} W+\frac{k_{B} T}{I} \frac{\partial W}{\partial \omega_{x}}\right)\right. \\
& +\beta \frac{\partial}{\partial \omega_{y}}\left(\omega_{y} W+\frac{k_{B} T}{I} \frac{\partial W}{\partial \omega_{y}}\right)+\beta_{z} \frac{\partial}{\partial \omega_{z}} \\
& \left.\times\left(\omega_{z} W+\frac{k_{B} T}{I_{z}} \frac{\partial W}{\partial \omega_{z}}\right)\right],
\end{aligned}
$$

where $\beta=\zeta / I, \beta_{z}=\zeta_{z} / I_{z}, \zeta$, and $\zeta_{z}$ are the viscous damping coefficients, $k_{B} T$ is the thermal energy, $\tau$ is an intertrapping time scale (see below) which we identify with the Debye relaxation time $\zeta /\left(2 k_{B} T\right)$ for normal diffusion of symmetrical top molecules, and $\alpha$ is the exponent characterizing the anomalous diffusion process. The operator ${ }_{0} D_{t}^{1-\alpha}$ $\equiv(\partial / \partial t)_{0} D_{t}^{-\alpha}$ in Eq. (4) is defined in terms of the convolution (the Riemann-Liouville fractional integral definition) [17]

$$
{ }_{0} D_{t}^{-\alpha} W(, t)=\frac{1}{\Gamma(\alpha)} \int_{0}^{t} \frac{W\left(, t^{\prime}\right) d t^{\prime}}{\left(t-t^{\prime}\right)^{1-\alpha}}
$$

so that the fractional derivative is a type of memory function $[2,17]$ or stosszahlansatz for the Boltzmann equation $[18,19]$ underlying the FKKE. For $\alpha=1$, Eq. (4) reduces to the corresponding normal Fokker-Planck equation for inertia corrected rotational diffusion already considered (see, e.g., Ref. [15]). Here, the internal field effects are ignored, which means that the effects of long-range torques due to the interaction between the average moments and the Maxwell fields are not taken into account. Such effects may be ignored for dilute systems in first approximation. Thus, the results obtained here are relevant to situations where dipole-dipole interactions have been eliminated by extrapolation of data to infinite dilution.

\section{SOLUTION OF THE INERTIAL FRACTIONAL KLEIN-KRAMERS EQUATION}

Let us suppose that the uniform electric field $\mathbf{E}$ having been applied to the assembly of polar nonpolarizable symmetric top molecules at a time $t=-\infty$ so that equilibrium conditions prevail by the time $t=0$, is switched off at $t=0$. In addition it is supposed that the field is weak (i.e., $\mu E$ $\ll k_{B} T$, which is the linear response condition). For $t>0$, the evolution of $W$ satisfies Eq. (4). Just as with $\alpha=1$, Eq. (4) is independent of the angles $\varphi$ and $\psi$ so that for the problem in question one may ignore the dependence of $W$ on $\varphi$ and $\psi$. Thus, we seek a solution of Eq. (4) by using the method of separation of variables in the form of the series

$$
\begin{aligned}
W\left(\vartheta, \omega_{x}, \omega_{y}, \omega_{z}, t\right)= & \eta_{z} \eta^{2} e^{-\eta^{2}\left(\omega_{x}^{2}+\omega_{y}^{2}\right)-\eta_{z}^{2} \omega_{z}^{2}} \\
& \times \sum_{l=0}^{\infty} \sum_{m=-l}^{l} \sum_{n=0}^{\infty} \sum_{k=0}^{\infty} a_{n, k}^{l, m} b_{n, k}^{l, m}(t) s_{n, k}^{m} \\
& \times\left(\omega_{x}, \omega_{y}, \omega_{z}\right) P_{l}^{|m|}(\cos \vartheta)
\end{aligned}
$$

where

$$
b_{n, k}^{l, m}(t)=\left\langle P_{l}^{|m|}(\cos \vartheta) s_{n, k}^{m}\left(\omega_{x}, \omega_{y}, \omega_{z}\right)\right\rangle,
$$

$P_{l}^{|m|}(\cos \vartheta)$ are the associated Legendre functions [20], and the functions $s_{n, k}^{m}\left(\omega_{x}, \omega_{y}, \omega_{z}\right) \quad(l, n, k=0,1,2, \ldots ;-l \leqslant m$ $\leqslant l$ ) are expressed as finite series of products of Hermite polynomials $H_{n}(z)$ [20] in the components $\omega_{x}, \omega_{y}$ and $\omega_{z}$ of the angular velocity, viz.,

$$
\begin{aligned}
s_{n, k}^{2 m-M}\left(\omega_{x}, \omega_{y}, \omega_{z}\right)= & H_{k}\left(\eta_{z} \omega_{z}\right) \sum_{q=0}^{n} \frac{r_{2 m-M}(n, q)}{q !(n-q) !} \\
& \times H_{2 n-2 q+M-\varepsilon_{m}}\left(\eta \omega_{x}\right) H_{2 q+\varepsilon_{m}}\left(\eta \omega_{y}\right) .
\end{aligned}
$$

Here $\eta=\sqrt{I /\left(2 k_{B} T\right)}, \quad \eta_{z}=\sqrt{I_{z} /\left(2 k_{B} T\right)}, \quad \varepsilon_{m}=0$ for $m \geqslant 0$, $\varepsilon_{m}=1$ for $m<0, \quad M=0$ or 1 and the coefficients $r_{2 m+M}(n, q)$ of the finite series are determined by the recurrence relations [16] 


$$
\begin{aligned}
r_{2 p}(n, q)= & \left(n-q+\frac{1}{2}\right)\left(1-\frac{2 q+1}{2 p-1}\right) r_{2 p-1}(n, q) \\
+ & (n-q) \frac{2 q+1}{2 p-1} r_{2 p-1}(n, q+1), \\
r_{2 p+1}(n, q)= & \left(1+\frac{q}{p}\right) r_{2 p}(n, q)-\frac{q}{p} r_{2 p}(n, q-1), \\
r_{-2 p}(n, q)= & (n-q)\left[\left(1-\frac{2 q+2}{2 p-1}\right) r_{-(2 p-1)}(n, q)\right. \\
& \left.+\frac{2 q+3}{2 p-1} r_{-(2 p-1)}(n, q+1)\right] \\
r_{-(2 p+1)}(n, q)= & \left(1+\frac{2 q+1}{2 p}\right) r_{-2 p}(n, q) \\
& -\frac{q}{2 p}\left(2-\frac{1}{n-q+1}\right) r_{-2 p}(n, q-1)
\end{aligned}
$$

with $r_{0}(n, q)=r_{ \pm 1}(n, q)=1$ and $p \geqslant 0$. The above recurrence relations and the orthogonality of the Hermite polynomials ensure that the functions $s_{n, k}^{m}\left(\omega_{x}, \omega_{y}, \omega_{z}\right)$ are orthogonal, viz.,

$$
\begin{aligned}
& \int_{-\infty}^{\infty} \int_{-\infty}^{\infty} \int_{-\infty}^{\infty} s_{n, k}^{m}\left(\omega_{x}, \omega_{y}, \omega_{z}\right) s_{n^{\prime}, k^{\prime}}^{m^{\prime}}\left(\omega_{x}, \omega_{y}, \omega_{z}\right) \\
& \times e^{-\eta^{2}\left(\omega_{x}^{2}+\omega_{y}^{2}\right)-\eta_{z}^{2} \omega_{z}^{2}} d \omega_{x} d \omega_{y} d \omega_{z} \sim \delta_{n, n^{\prime}} \delta_{m, m^{\prime}} \delta_{k, k^{\prime}},
\end{aligned}
$$

and that they form a complete set in angular velocity space. The angular brackets denote ensemble averages over the distribution function $W$, viz.,

$$
\begin{aligned}
\langle A\rangle(t)= & \int_{-\infty}^{\infty} \int_{-\infty}^{\infty} \int_{-\infty}^{\infty} \int_{0}^{\pi} A\left(\vartheta, \omega_{x}, \omega_{y}, \omega_{z}\right) \\
& \times W\left(\vartheta, \omega_{x}, \omega_{y}, \omega_{z}, t\right) \sin \vartheta d \vartheta d \omega_{x} d \omega_{y} d \omega_{z}
\end{aligned}
$$

The normalizing constants $a_{n, k}^{l, m}$ are obtained by means of the orthogonality properties of the $P_{l}^{m}(\cos \vartheta)$ and $H_{n}(z)$, viz. [20],

$$
\begin{gathered}
\int_{0}^{\pi} P_{l}^{m}(\cos \vartheta) P_{l^{\prime}}^{m^{\prime}}(\cos \vartheta) \sin \vartheta d \vartheta \\
=\frac{2(l+m) !}{(2 l+1)(l-m) !} \delta_{l, l^{\prime}} \delta_{m, m^{\prime}}, \\
\int_{-\infty}^{\infty} H_{n}(x) H_{n^{\prime}}(x) e^{-x^{2}} d x=\sqrt{\pi} 2^{n} n ! \delta_{n, n^{\prime}}
\end{gathered}
$$

so that

$$
\begin{aligned}
\left(a_{n, k}^{l, 2 m-M}\right)^{-1}= & \frac{\pi^{3 / 2} 2^{2 n+k+M+1} k !(l+m) !}{(2 l+1)(l-m) !} \\
& \times \sum_{q=0}^{n}\left(\frac{r_{2 m-M}(n, q)}{q !(n-q) !}\right)^{2} \\
& \times\left(2 n-2 q+M-\varepsilon_{m}\right) !\left(2 q+\varepsilon_{m}\right) ! .
\end{aligned}
$$

Substituting Eq. (6) into Eq. (4), taking the inner product, and utilizing the orthogonal properties and known recurrence relations [20] for the associated Legendre functions and the Hermite polynomials, then yields the infinite hierarchy of differential recurrence relations for the $b_{n, k}^{l, m}(t)$ governing the orientational relaxation of the system, viz.,

$$
\begin{aligned}
& \eta \frac{d}{d t} b_{n, k}^{l, 0}=-\left[2 n \beta^{\prime}+k \beta_{z}^{\prime} / B\right] \tau^{1-\alpha}{ }_{0} D_{t}^{1-\alpha} b_{n, k}^{l, 0} \\
& +\frac{1}{2} b_{n, k}^{l, 1}+2 b_{n-1, k}^{l, 1} \\
& \eta \frac{d}{d t} b_{n, k}^{l, \pm 1}=-\left[(2 n+1) \beta^{\prime}+k \beta_{z}^{\prime} / B\right] \tau^{1-\alpha}{ }_{0} D_{t}^{1-\alpha} b_{n, k}^{l, \pm 1} \\
& +b_{n, k}^{l, \pm 2}+\frac{1}{4} b_{n+1, k}^{l, \pm 2}-\left(1-\delta_{ \pm 1,-1}\right) l(l+1)(n+1) \\
& \times\left[b_{n, k}^{l, 0}+\frac{1}{4} b_{n+1, k}^{l, 0}\right] \mp \sqrt{B}\left(\frac{1}{2} b_{n, k+1}^{l, \mp 1}+k b_{n, k-1}^{l, \mp 1}\right), \\
& \eta \frac{d}{d t} b_{n, k}^{l, \pm 2}=-\left[2 n \beta^{\prime}+k \beta_{z}^{\prime} / B\right]_{0} D_{t}^{1-\alpha} \tau^{1-\alpha} b_{n, k}^{l, \pm 2}+b_{n-1, k}^{l, \pm 3} \\
& +\frac{1}{4} b_{n, k}^{l, \pm 3}-(l+2)(l-1)\left[(n+1) b_{n-1, k}^{l, \pm 1}\right. \\
& \left.+\frac{n}{4} b_{n, k}^{l, \pm 1}\right] \mp 2 \sqrt{B}\left(\frac{1}{2} b_{n, k+1}^{l, \mp 2}+k b_{n, k-1}^{l, \mp 2}\right),
\end{aligned}
$$

and so on. Here $\beta^{\prime}=\eta \zeta / I, \beta_{z}^{\prime}=\eta \zeta_{z} / I$, and $B=I_{z} / I$. For linear molecules $\left(B=0, \beta_{z}^{\prime} / B \rightarrow 0\right)$, Eqs. (17), (18) yield the results of Ref. [11].

\section{DIELECTRIC RELAXATION OF AN ENSEMBLE OF SYMMETRIC TOP MOLECULES}

In dielectric relaxation, $l=1$ so that by taking the Laplace transform of Eqs. (17)-(19) over the time variables and noting the generalized integral theorem for Laplace transforms $[17,21,22]$, namely,

$$
L\left\{{ }_{0} D_{t}^{1-\alpha} f(t)\right\}= \begin{cases}s^{1-\alpha} \widetilde{f}(s)-\left.{ }_{0} D_{t}^{-\alpha} f(t)\right|_{t=0} & (0<\alpha<1), \\ s^{1-\alpha} \widetilde{f}(s) & (1 \leqslant \alpha<2),\end{cases}
$$

where 


$$
\widetilde{f}(s)=L\{f(t)\}=\int_{0}^{\infty} e^{-s t} f(t) d t,
$$

we then have a system of algebraic recurrence relations for the Laplace transform of $b_{n, k}^{1, m}(t) \quad(m=0, \pm 1)$ [so that $\left.b_{0,0}^{1,0}(t) \equiv\langle\cos \theta\rangle(t)\right]$ governing the dielectric response. These equations can be written as a system of algebraic recurrence relations in the frequency domain using Laplace transformation, namely,

$$
\begin{aligned}
{[\eta s+} & \left.2 n \rho(s)+k \rho_{z}(s) / B\right] \widetilde{b}_{n, k}^{1,0} \\
= & \eta b_{0,0}^{1,0}(0) \delta_{n+k, 0}+\frac{1}{2} \widetilde{b}_{n, k}^{1,1}+2 \widetilde{b}_{n-1, k}^{1,1}, \\
{[\eta s+} & \left.(2 n+1) \rho(s)+k \rho_{z}(s) / B\right] \widetilde{b}_{n, k}^{1,1} \\
= & -\frac{1}{2}(n+1) \widetilde{b}_{n+1, k}^{1,0}-2(n+1) \widetilde{b}_{n, k}^{1,0} \\
& -\frac{1}{2} \sqrt{B}\left(\widetilde{b}_{n, k+1}^{1,-1}+2 k \widetilde{b}_{n, k-1}^{1,-1}\right) \\
{[\eta s+} & \left.(2 n+1) \rho(s)+k \rho_{z}(s) / B\right] \widetilde{b}_{n, k}^{1,-1} \\
= & \frac{1}{2} \sqrt{B}\left(\widetilde{b}_{n, k+1}^{1,1}+2 k \widetilde{b}_{n, k-1}^{1,1}\right) .
\end{aligned}
$$

Here

$$
\rho(s)=\beta^{\prime}(\tau s)^{1-\alpha}, \quad \rho_{z}(s)=\beta_{z}^{\prime}(\tau s)^{1-\alpha}
$$

and we have noted that all the $b_{n, k}^{1,0}(0)$ vanish with the exception of $n+k=0$, viz., $\quad b_{0,0}^{1,0}(0)=\xi / 3$, where $\xi$ $=\mu E /\left(k_{B} T\right)$. This initial condition follows from the linearized initial (at $t=0$ ) distribution function, which has the Maxwell-Boltzmann form

$$
\begin{aligned}
W\left(\vartheta, \omega_{x}, \omega_{y}, 0\right)= & \frac{\eta_{z} \eta^{2}}{2 \pi^{3 / 2}} e^{-\eta^{2}\left(\omega_{x}^{2}+\omega_{x}^{2}\right)-\eta_{z}^{2} \omega_{z}^{2}} \\
& \times\left(1+\xi \cos \vartheta+\frac{\xi^{2}}{2} \cos ^{2} \vartheta\right)+o\left(\xi^{2}\right) .
\end{aligned}
$$

In order to solve the hierarchy of recurrence Eqs. (21)-(23), we introduce a supercolumn vector $\widetilde{\mathbf{C}}_{n}(s)$ comprising three subvectors:

$$
\widetilde{\mathbf{C}}_{n}(s)=\left(\begin{array}{c}
\widetilde{\mathbf{c}}_{n-1}^{1,0}(s) \\
\widetilde{\mathbf{c}}_{n-1}^{1,1}(s) \\
\widetilde{\mathbf{c}}_{n-1}^{1,-1}(s)
\end{array}\right), \quad \widetilde{\mathbf{c}}_{n}^{1, m}(s)=\left(\begin{array}{c}
\widetilde{b}_{n, 0}^{1, m}(s) \\
\widetilde{b}_{n-1,1}^{1, m}(s) \\
\vdots \\
\widetilde{b}_{0, n}^{1, m}(s)
\end{array}\right) .
$$

The subvector $\tilde{\mathbf{c}}_{n}^{1, m}(s)$ has the dimension $n+1$. The three index recurrence Eqs. (21)-(23) for $\widetilde{b}_{n, k}^{1, m}(s)$ can then be transformed into the matrix three-term differentialrecurrence equation

$$
\begin{aligned}
& {\left[\eta s \mathbf{I}_{3 n}-\mathbf{Q}_{n}(s)\right] \widetilde{\mathbf{C}}_{n}(s)-\mathbf{Q}_{n}^{+} \widetilde{\mathbf{C}}_{n+1}(s)-\mathbf{Q}_{n}^{-} \widetilde{\mathbf{C}}_{n-1}(s)} \\
& \quad=\delta_{n, 1} \eta \mathbf{C}_{1}(0) \quad(n \geqslant 1)
\end{aligned}
$$

where

$$
\begin{gathered}
\mathbf{Q}_{n}^{-=}\left(\begin{array}{ccc}
\mathbf{0} & \mathbf{q}_{n}^{-} & \mathbf{0} \\
\mathbf{0} & \mathbf{0} & -\mathbf{p}_{n}^{-} \\
\mathbf{0} & \mathbf{p}_{n}^{-} & \mathbf{0}
\end{array}\right), \quad \mathbf{Q}_{n}^{+}=\left(\begin{array}{ccc}
\mathbf{0} & \mathbf{0} & \mathbf{0} \\
\mathbf{q}_{n}^{+} & \mathbf{0} & -\mathbf{p}_{n}^{+} \\
\mathbf{0} & \mathbf{p}_{n}^{+} & \mathbf{0}
\end{array}\right), \\
\mathbf{Q}_{n}(s)=\left(\begin{array}{ccc}
\mathbf{q}_{n}^{0}(s) & \mathbf{I}_{n} / 2 & \mathbf{0} \\
2\left(\mathbf{r}_{n}-\mathbf{I}_{n}\right) & \mathbf{q}_{n}^{1}(s) & \mathbf{0} \\
\mathbf{0} & \mathbf{0} & \mathbf{q}_{n}^{1}(s)
\end{array}\right),
\end{gathered}
$$

and

$$
\mathbf{q}_{n}^{-}=2\left(\begin{array}{cccc}
1 & 0 & \cdots & 0 \\
0 & 1 & \cdots & 0 \\
\vdots & \vdots & \ddots & \vdots \\
0 & 0 & \cdots & 1 \\
0 & 0 & \cdots & 0
\end{array}\right)_{n \times(n-1)},
$$

$$
\mathbf{q}_{n}^{+}=-\frac{1}{2}\left(\begin{array}{ccccc}
n & 0 & \cdots & 0 & 0 \\
0 & n-1 & \cdots & 0 & 0 \\
\vdots & \vdots & \ddots & \vdots & \vdots \\
0 & 0 & \cdots & 1 & 0
\end{array}\right)_{n \times(n+1)},
$$$$
\mathbf{p}_{n}^{-}=\sqrt{B}\left(\begin{array}{cccc}
0 & \cdots & 0 & 0 \\
1 & \cdots & 0 & 0 \\
\vdots & \ddots & \vdots & \vdots \\
0 & \cdots & n-2 & 0 \\
0 & \cdots & 0 & n-1
\end{array}\right)_{n \times(n-1)}
$$

$$
\mathbf{p}_{n}^{+}=\frac{\sqrt{B}}{2}\left(\begin{array}{ccccc}
0 & 1 & \cdots & 0 & 0 \\
\vdots & \vdots & \ddots & \vdots & \vdots \\
0 & 0 & \cdots & 1 & 0 \\
0 & 0 & \cdots & 0 & 1
\end{array}\right)_{n \times(n+1)},
$$

$$
\mathbf{r}_{n}=-\left(\begin{array}{cccc}
n-1 & 0 & \cdots & 0 \\
0 & n-2 & \cdots & 0 \\
\vdots & \vdots & \ddots & \vdots \\
0 & 0 & \cdots & 0
\end{array}\right)_{n \times n}
$$




$$
\mathbf{q}_{n}^{M}(s)=\left(\begin{array}{cccc}
f_{n-1,0}^{M}(s) & 0 & \cdots & 0 \\
0 & f_{n-2,1}^{M}(s) & \cdots & 0 \\
\vdots & \vdots & \ddots & \vdots \\
0 & 0 & \cdots & f_{0, n-1}^{M}(s)
\end{array}\right)_{n \times n} \quad \mathbf{C}_{1}(0)=\left(\begin{array}{c}
\xi / 3 \\
0 \\
0
\end{array}\right)
$$

Here $f_{n, k}^{M}(s)=-(2 n+M) \rho(s)-k \rho_{z}(s) / B$ and $\mathbf{I}_{n}$ is the unit and $\mathbf{C}_{n}(0)=\mathbf{0}$ for all $n \geqslant 2$. The exact solution for $\widetilde{\mathbf{C}}_{1}(s)$ is matrix which has dimension $n \times n$. The initial value vector

$$
\widetilde{\mathbf{C}}_{1}(s)=\eta \frac{\mathbf{I}_{3}}{\eta s \mathbf{I}_{3}-\mathbf{Q}_{1}(s)-\mathbf{Q}_{1}^{+} \frac{\mathbf{I}_{6}}{\eta s \mathbf{I}_{6}-\mathbf{Q}_{2}(s)-\mathbf{Q}_{2}^{+} \frac{\mathbf{I}_{9}}{\eta s \mathbf{I}_{9}-\mathbf{Q}_{3}(s)^{\cdot} \cdot} \mathbf{Q}_{3}^{-}} \mathbf{Q}_{2}^{-}} \mathbf{C}_{1}(0)
$$

where the fraction lines denote matrix inversion. Having determined the spectrum $\widetilde{b}_{0,0}^{1,0}(i \omega)$ from Eq. (28), one can also evaluate the normalized complex susceptibility $\hat{\chi}(\omega)$ $=\hat{\chi}^{\prime}(\omega)-i \hat{\chi}^{\prime \prime}(\omega)$ from linear response theory as (Ref. [23], p. 203)

$$
\hat{\chi}(\omega)=\frac{\chi(\omega)}{\chi^{\prime}(0)}=1-i \omega \frac{\widetilde{b}_{0,0}^{1,0}(i \omega)}{b_{0,0}^{1,0}(0)},
$$

where $\chi^{\prime}(0)=N_{0} \mu^{2} / 3 k_{B} T$ is the static susceptibility and $N_{0}$ is the number of dipoles per unit volume.

For linear and spherical top molecules, the solution can be considerably simplified and presented as ordinary continued fractions. For linear molecules $\left(I_{z}=0\right)$, such a solution was obtained in Ref. [11]. For spherical tops $\left(\beta^{\prime}=\beta_{z}^{\prime}\right.$, and $B$ $=1$ ), the corresponding solution is (see Appendix A)

$$
\frac{\tilde{b}_{0,0}^{1,0}(s)}{b_{0,0}^{1,0}(0)}=\frac{\eta}{s \eta+\frac{1}{s \eta+\rho(s)+\frac{1}{4[s \eta+2 \rho(s)]}+\frac{(3-1 / 2) / 2}{s \eta+2 \rho(s)+\frac{2}{s \eta+3 \rho(s)+\frac{1}{6[s \eta+4 \rho(s)]}+\frac{(5-1 / 3) / 2}{s \eta+4 \rho(s)+\cdots}}}}}
$$

For $\alpha=1, \rho(s)=\beta^{\prime}$ and Eq. (30) coincides with that of Sack [14] for normal rotational diffusion with a corrected misprint [16]. In the high damping limit $\left(\beta^{\prime} \gg 1\right)$, Eq. (30) can be simplified yielding the generalization to fractional dynamics of the Rocard [16] equation, namely,

$$
\hat{\chi}(\omega)=\frac{1}{1+(i \omega \tau)^{\sigma}-(\omega \eta)^{2}},
$$

where

$$
\sigma=2-\alpha
$$

On neglecting inertial effects $(\eta \rightarrow 0)$, Eq. (31) reduces to Eq. (1), i.e., the result previously proposed from empirical considerations $[24,25]$. The results of numerical calculations indicate that the matrix continued fraction solution [Eqs. (28) and (29)] and the ordinary continued fraction solution of Ref. [11] for linear molecules and Eq. (30) for spherical tops yield the same results.

The approach we have developed may also be extended to treat all the other averages $\left\langle P_{n}(\cos \vartheta)\right\rangle(t)$ characterizing orientational relaxation in fluids $[2,3]$, in particular, to evaluate the average of the second-order Legendre polynomial $\left\langle P_{2}(\cos \vartheta)\right\rangle(t)$ (this quantity describes the dynamic Kerreffect) which is given in Appendix B.

\section{RESULTS AND DISCUSSION}

The infinite matrix continued fraction Eq. (28) is easily computed so that the complex dielectric susceptibility Eq. 


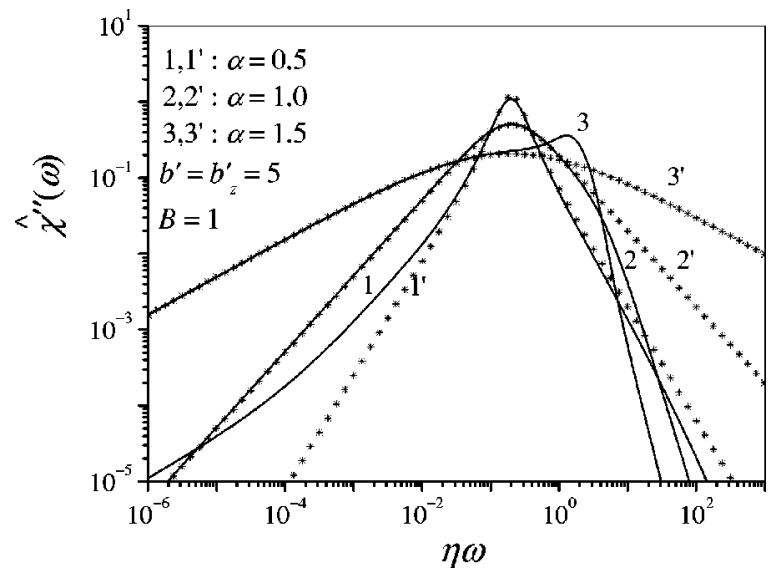

FIG. 1. Dielectric loss spectra $\hat{\chi}^{\prime \prime}(\omega)$ for $\beta^{\prime}=\beta_{z}^{\prime}=5$ and various values of $\alpha: \alpha=0.5$ (curves 1 and $1^{\prime}$ ), $\alpha=1.0$ (curves 2 and $2^{\prime}$ ), and $\alpha=1.5$ (curves 3 and $3^{\prime}$ ). Solid lines (1, 2, and 3): Eqs. (28) and (29); asterisks $\left(1^{\prime}, 2^{\prime}\right.$, and $\left.3^{\prime}\right)$ : Eq. (1).

(29) can be readily evaluated for typical values of the model parameters $\eta, \beta^{\prime}, \beta_{z}^{\prime}, B$, and $\alpha$. Dielectric loss $\hat{\chi}^{\prime \prime}(\omega)$ spectra for various values of $\alpha, \beta^{\prime}, \beta_{z}^{\prime}$ and $B$ are shown in Figs. $1-5$. It is apparent that the half-width and the shape of the dielectric spectra strongly depend on $\alpha$ (here pertaining to anomalous diffusion in velocity space), $\beta^{\prime}, \beta_{z}^{\prime}$, (which characterize the effects of damping and molecular inertia), and $B$ (which accounts for the shape of the molecule). For high damping, $\beta^{\prime}, \beta_{z}^{\prime} \gg 1$, the low frequency part of $\hat{\chi}^{\prime \prime}(\omega)$ may be approximated by the Cole-Cole Eq. (1) (see Figs. 1-5). On the other hand, the high-frequency behavior of $\hat{\chi}^{\prime \prime}(\omega)$ is entirely determined by the inertia of system. Just as in normal Brownian dynamics, it is apparent that inertial effects produce a much more rapid falloff of $\hat{\chi}^{\prime \prime}(\omega)$ at high frequencies. Indeed, one can show that our fractional model satisfies the Gordon sum rule for the dipole integral absorption [26], viz.,

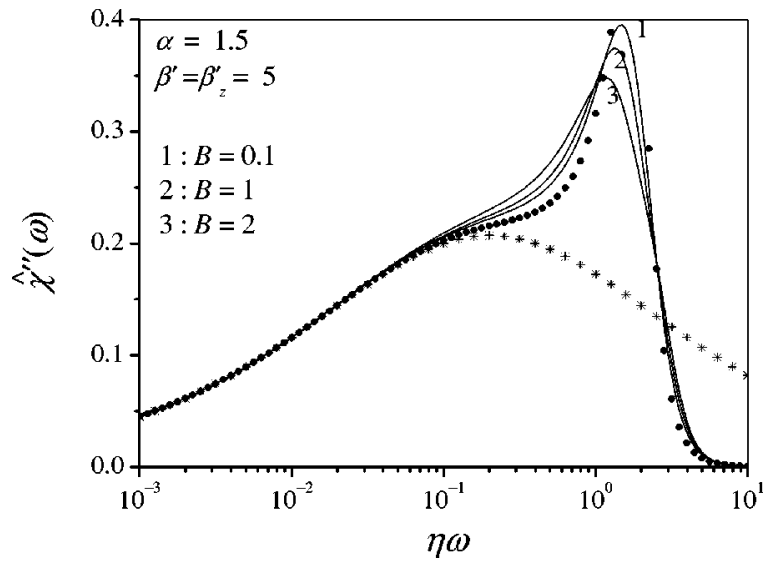

FIG. 2. Dielectric loss spectra $\hat{\chi}^{\prime \prime}(\omega)$ for $\alpha=1.5, \beta^{\prime}=\beta_{z}^{\prime}=5$ and various values of $B: B=0.1$ (curve 1), $B=1$ (curve 2), $B=2$ (curve 3); circles: Eq. (31); asterisks: Eq. (1).

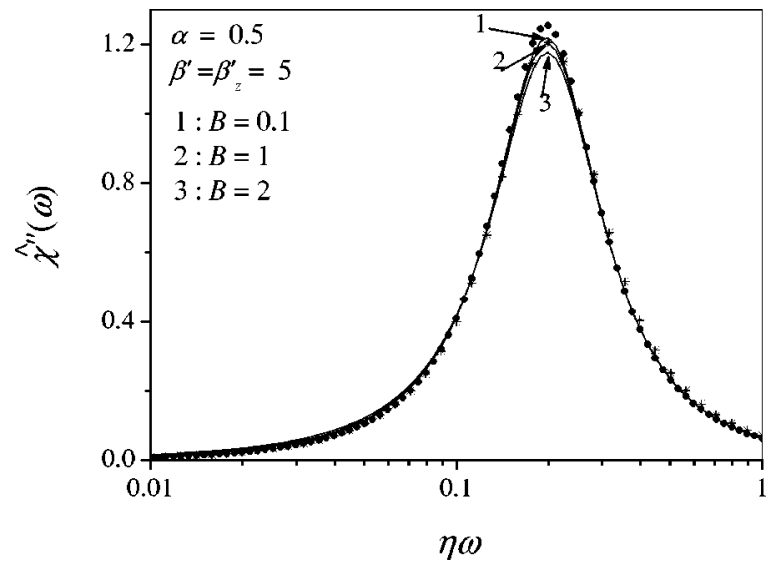

FIG. 3. Dielectric loss spectra $\hat{\chi}^{\prime \prime}(\omega)$ for $\alpha=0.5, \beta^{\prime}=\beta_{z}^{\prime}=5$ and various values of $B: B=0.1$ (curve 1 ), $B=1$ (curve 2), $B=2$ (curve 3); circles: Eq. (31); asterisks: Eq. (1).

$$
\int_{0}^{\infty} \omega \chi^{\prime \prime}(\omega) d \omega=\frac{\pi N \mu^{2}}{3 I}
$$

It is significant that the right hand side of Eq. (33) is determined by molecular parameters only and is independent of the temperature and the model parameters $\alpha, \beta^{\prime}$, and $\beta_{z}^{\prime}$. In contrast, the fractional noninertial rotational diffusion model [3] [yielding Eq. (1)] predicts infinite integral absorption.

We remark that all the above results are obtained by using the Barkai-Silbey [9] fractional form of the Klein-Kramers equation for the evolution of the probability distribution function in phase space. In that equation, the fractional derivative, or memory term, acts only on the right-hand side, that is, on the diffusion or dissipative term. Thus, the form of the Liouville operator, or convective derivative is preserved [cf. the right-hand side of Eq. (4)]. Thus, Eq. (4) has the conventional form of a Boltzmann equation for the single particle distribution function. The preservation of the Liou-

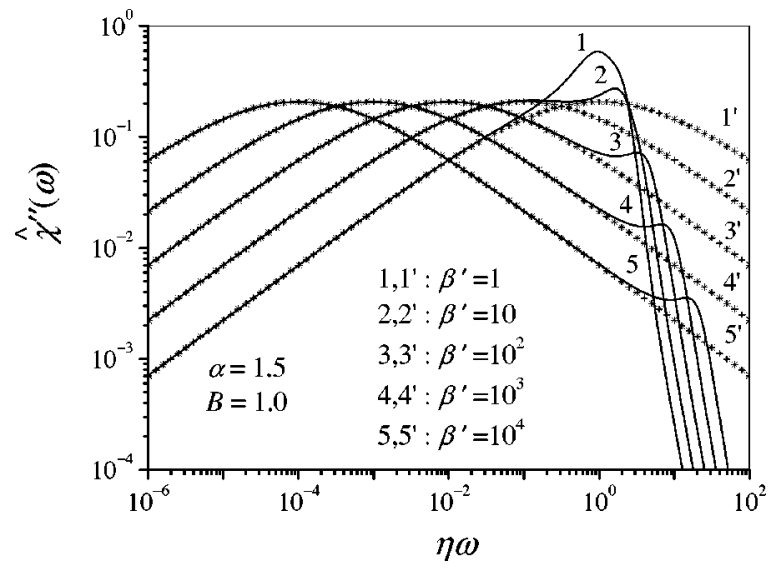

FIG. 4. Dielectric loss spectra $\hat{\chi}^{\prime \prime}(\omega)$ for $\alpha=1.5, B=1$ and various values of $\beta^{\prime}=\beta_{z}^{\prime}: \beta^{\prime}=1$ (curve 1), $\beta^{\prime}=10$ (curve 2), $B$ $=100$ (curve 3$), \beta^{\prime}=10^{3}$ (curve 4$)$, and $\beta^{\prime}=10^{4}$ (curve 5). Asterisks: Eq. (1). 


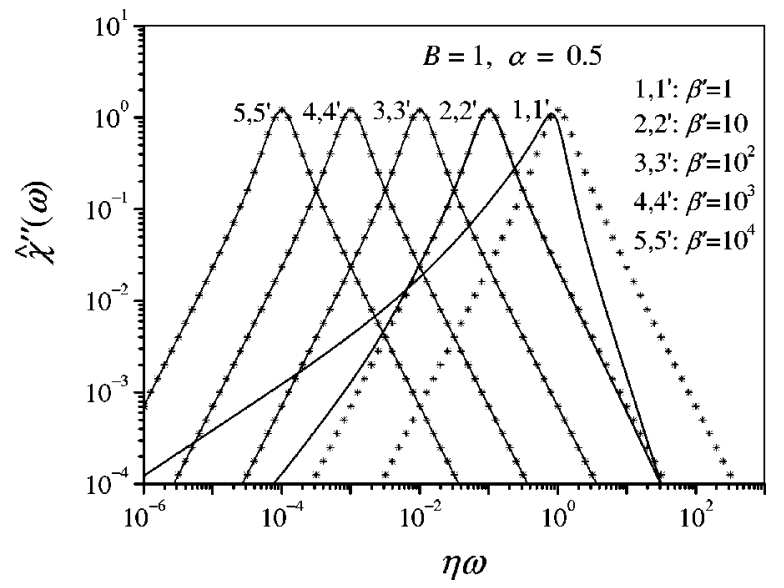

FIG. 5. Dielectric loss spectra $\hat{\chi}^{\prime \prime}(\omega)$ for $\alpha=0.5, B=1$ and various values of $\beta^{\prime}=\beta_{z}^{\prime}: \beta^{\prime}=1$ (curve 1), $\beta^{\prime}=10$ (curve 2), $\beta^{\prime}$ $=100$ (curve 3$), \beta^{\prime}=10^{3}$ (curve 4$)$, and $\beta^{\prime}=10^{4}$ (curve 5). Asterisks: Eq. (1).

ville operator is equivalent to stating that the Newtonian form of the equations of motion underlying the KleinKramers equation is preserved. Thus, the high frequency behavior is entirely controlled by the inertia of the system, and does not depend on the anomalous exponent. Consequently, the fundamental sum rule, Eq. (33), for the dipole integral absorption of single axis rotators is satisfied, ensuring a return to transparency at high frequencies as demanded on physical grounds.

At this stage, it is appropriate to mention yet another generalization to fractional dynamics of the Klein-Kramers equation, which has been proposed in Ref. [7]. Here, unlike Eq. (4), the fractional derivative acts on the convective as well as the diffusive terms in the normal Klein-Kramers equation. This equation, in the notation of Eq. (4), reads

$$
\begin{aligned}
\frac{\partial W}{\partial t}= & \tau^{1-\alpha} D_{t}^{1-\alpha}\left\{-\omega_{x} \frac{\partial W}{\partial \vartheta}-\left(\omega_{y} \cot \vartheta-\frac{I_{z}}{I} \omega_{z}\right)\right. \\
& \times\left(\omega_{y} \frac{\partial W}{\partial \omega_{x}}-\omega_{x} \frac{\partial W}{\partial \omega_{y}}\right)+\left[\beta \frac{\partial}{\partial \omega_{x}}\left(\omega_{x} W+\frac{k_{B} T}{I} \frac{\partial W}{\partial \omega_{x}}\right)\right. \\
& +\beta \frac{\partial}{\partial \omega_{y}}\left(\omega_{y} W+\frac{k_{B} T}{I} \frac{\partial W}{\partial \omega_{y}}\right)+\beta_{z} \frac{\partial}{\partial \omega_{z}} \\
& \left.\left.\times\left(\omega_{z} W+\frac{k_{B} T}{I_{z}} \frac{\partial W}{\partial \omega_{z}}\right)\right]\right\} .
\end{aligned}
$$

Equation (34) may also be solved exactly in terms of a scalar continued fraction just as Eq. (4) for the complex susceptibility of an assembly of noninteracting dipoles. However, the complex susceptibility so rendered does not satisfy the Gordon sum rule, the absorption coefficient $\omega \chi^{\prime \prime}(\omega)$ showing a marked divergence at high frequencies as $\omega$ increases. Thus, the high frequency behavior of the dielectric susceptibility predicted by Eq. (34), is physically unacceptable, unlike that predicted by the Barkai-Silbey equation. For example, the generalization of the Rocard equation gained using Eq. (34), viz.,

$$
\hat{\chi}(\omega)=\frac{1}{1+(i \omega \tau)^{\alpha}+\beta^{\prime-2}(i \omega \tau)^{2 \alpha}},
$$

predicts a divergent absorption coefficient. The root of the problem appears to be the supposition that the fractional derivative (or memory function with a power law kernel so that the process depends on the history of the system) acts on the convective terms. If it is allowed to act on these terms, then the high frequency behavior is no longer entirely inertia controlled, it depends on the dissipation through the anomalous exponent, with the result, that the Gordon sum rules are violated, and infinite absorption ensues. This is the reason for the use of the Barkai-Silbey equation. We also remark that the Barkai-Silbey equation was originally given for subdiffusion in velocity space $(\alpha<1)$, or for enhanced diffusion in configuration space $(\sigma>1)$. However, the most interesting case is subdiffusion in configuration space, corresponding to the Cole-Cole equation in dielectric theory. This suggests extending the Barkai-Silbey equation to enhanced diffusion in velocity space, corresponding to subdiffusion in configuration space, since $\sigma=2-\alpha$. The justification for doing this is simply that this generalization yields physically meaningful results for the broad-band spectrum of the complex susceptibility $\hat{\chi}(\omega)$, as well as yielding the Cole-Cole equation in the limit $\gamma \rightarrow 0$. It is also apparent that the Barkai-Silbey equation must have its origin in a Lévy (rather than a purely fractal) time random walk [7], as unlike Eq. (34), it does not separate into temporal and spatial parts, moreover, the exponential decay of the normal diffusion theory is not replaced by a Mittag-Leffler function, as in a fractal time random walk [7]. Such behavior is indicative of coupling between the jump length probability distribution and the waiting time probability distribution, that is, the jump length and waiting time are not independent random variables.

As far as comparison with experimental data is concerned, the fractional Klein-Kramers model under discussion may be suitable for the explanation of dielectric relaxation of dilute solution of polar molecules (such as $\mathrm{CHCl}_{3}, \mathrm{CH}_{3} \mathrm{Cl}$, etc.) in nonpolar glassy solvents (such as decalin at low temperatures, see, e.g., Ref. [27]). Here, in contrast to the normal diffusion, the model can explain qualitatively the inertiacorrected anomalous (Cole-Cole-like) dielectric relaxation behavior of such solutions at low frequencies. However, one would expect that the model is not applicable at high frequencies (in the far-infrared region), where the librational character of the rotational motion must be taken into account. The failure of the fractional Klein-Kramers model to account for the high-frequency (Poley) absorption even though it explains the return to transparency at high frequencies is to be expected in view of the assumption made in the 
theory that all electrical interactions between dipoles may be neglected.

\section{ACKNOWLEDGMENTS}

The support of this work by USAF, EOARD (Contract No. FA8655-03-01-3027), the Enterprise Ireland-CNRS France scheme 2003, and the Russian Foundation for Basic Research (Project No. 01-02-16050) is gratefully acknowledged.

\section{APPENDIX A: ORDINARY CONTINUED FRACTION SOLUTION FOR SPHERICAL TOP MOLECULES}

A solution of the system of moment equations (21)-(23) can be obtained in terms of ordinary continued fractions for spherical top molecules $\left(\beta^{\prime}=\beta_{z}^{\prime}\right.$, and $\left.B=1\right)$ as follows. On substituting Eqs. (21) and (23) into Eq. (22) and introducing a new quantity $a_{n, k}$ defined as $a_{n, k}=\widetilde{b}_{n, 2 k}^{1,1} / k$ !, we obtain

$$
\begin{aligned}
{[\eta s+} & {\left.[2(n+k)+1] \rho(s)+\frac{2(n+k)+3}{2\{\eta s+[2(n+k)+2] \rho(s)\}}+\frac{2(n+k)+2}{2[\eta s+2(n+k) \rho(s)]}\right] a_{n, k} } \\
= & -\frac{2}{s} b_{0,0}^{1,0}(0) \delta_{n+k, 0}-\frac{n+1}{4\{\eta s+[2(n+k)+2] \rho(s)\}} a_{n+1, k}-\frac{k+1}{4\{\eta s+[2(n+k)+2] \rho(s)\}} a_{n, k+1} \\
& -\frac{4(n+1)}{\eta s+2(n+k) \rho(s)} a_{n-1, k}-\frac{4 k-2}{\eta s+2(n+k) \rho(s)} a_{n, k-1} .
\end{aligned}
$$

Further, for the series

$$
S_{m}=\sum_{i=0}^{m} a_{m-i, i}
$$

(where the summation is taken over the elements $a_{n, k}$ with $n+k=m$, e.g., $S_{0}=a_{0,0}, S_{1}=a_{1,0}+a_{0,1}, S_{2}=a_{2,0}+a_{1,1}+a_{0,2}$, etc.), we have from Eqs. (A1) and (A2)

$$
\begin{aligned}
{[\eta s} & \left.+(2 m+1) \rho(s)+\frac{2 m+3}{2[\eta s+2(m+1) \rho(s)]}+\frac{2 m+2}{2[\eta s+2 m \rho(s)]}\right] S_{m} \\
& =-\frac{2}{s} b_{0,0}^{1,0}(0) \delta_{m, 0}-\frac{m+1}{4[\eta s+2(m+1) \rho(s)]} S_{m+1}-\frac{4 m+6}{\eta s+2 m \rho(s)} S_{m-1}
\end{aligned}
$$

or

$$
\left(\eta s-q_{m}\right) S_{m}-q_{m}^{+} S_{m+1}-q_{m}^{-} S_{m-1}=-\frac{2}{s} b_{0,0}^{1,0}(0) \delta_{m, 0},
$$

where

$$
\begin{gathered}
q_{m}=-(2 m+1) \rho(s)-\frac{2 m+3}{2[\eta s+2(m+1) \rho(s)]} \\
-\frac{m+1}{\eta s+2 m \rho(s)}, \\
q_{m}^{+}=-\frac{m+1}{4[\eta s+2(m+1) \rho(s)]}, \\
q_{m}^{-}=-\frac{4 m+6}{\eta s+2 m \rho(s)} .
\end{gathered}
$$

The continued fraction solution of the three-term recurrence Eq. (A4) is

$$
S_{0}=-\frac{2}{\eta s} \frac{\eta b_{0,0}^{1,0}(0)}{\eta s-q_{0}-\frac{q_{0}^{+} q_{1}^{-}}{\eta s-q_{1}-\frac{q_{1}^{+} q_{2}^{-}}{\eta s-q_{2}-\cdot}}} .
$$

The quantity $\widetilde{b}_{0,0}^{1,0}$ is then given by

$$
\widetilde{b}_{0,0}^{1,0}=\frac{b_{0,0}^{1,0}(0)}{s}+\frac{1}{2 \eta s} S_{0} .
$$

Taking into account Eqs. (A4)-(A9) and noting the equality 


$$
\begin{gathered}
\frac{2 n+3}{2 A}-\frac{(n+1)(2 n+5)}{2 A^{2}} \frac{1}{\frac{n+2}{A}+B} \\
=\frac{1}{2(n+2) A}+\frac{\frac{1}{2}\left(2 n+3-\frac{1}{n+2}\right)}{A+\frac{n+2}{B}},
\end{gathered}
$$

we obtain

$$
\frac{\widetilde{b}_{0,0}^{1,0}(s)}{b_{0,0}^{1,0}(0)}=\frac{\eta}{s \eta+Z_{2}}
$$

where the infinite continued fraction $Z_{2}$ is determined by the recurrence relation

$$
Z_{n}=\frac{n / 2}{s \eta+\rho(s)(n-1)+\frac{1}{(n+2)[s \eta+n \rho(s)]}+\frac{n(n+3)}{2(n+2)\left[s \eta+n \rho(s)+Z_{n+2}\right]}} .
$$

Equations (A10) and (A11) yield Eq. (30).

\section{APPENDIX B: DYNAMIC KERR EFFECT RESPONSE}

One may also readily derive differential-recurrence equations for the statistical moments involving the associate Legendre functions of order $2(l=2)$ pertaining to the dynamic Kerr effect, viz., $b_{n, k}^{2, m}(t)$ [so that $\left.b_{0,0}^{2,0}(t)=\left\langle P_{2}(\cos \vartheta)\right\rangle(t)\right]$. These equations can be written as a system of algebraic recurrence relations in the frequency domain using Laplace transformation, namely,

$$
\begin{gathered}
{\left[\eta s+2 n \rho(s)+k \rho_{z}(s) / B\right] \widetilde{b}_{n, k}^{2,0}} \\
=\eta c_{0,0}^{2,0}(0) \delta_{n+k, 0}+\frac{1}{2} \widetilde{b}_{n, k}^{2,1}+2 \widetilde{b}_{n-1, k}^{2,1}, \quad(\mathrm{~B} 1) \\
{\left[\eta s+(2 n+1) \rho(s)+k \rho_{z}(s) / B\right] \widetilde{b}_{n, k}^{2,1}} \\
=-\frac{3}{2}(n+1) \widetilde{b}_{n+1, k}^{2,0}-6(n+1) \widetilde{b}_{n, k}^{2,0}+\frac{1}{4} \widetilde{b}_{n+1, k}^{2,2}+\widetilde{b}_{n, k}^{2,2} \\
-\frac{1}{2} \sqrt{B}\left(\widetilde{b}_{n, k+1}^{2,-1}+2 k \widetilde{b}_{n, k-1}^{2,-1}\right), \\
{\left[\eta s+(2 n+1) \rho(s)+k \rho_{z}(s) / B\right] \widetilde{b}_{n, k}^{2,-1}} \\
=\frac{1}{4} \widetilde{b}_{n+1, k}^{2,-2}+\widetilde{b}_{n, k}^{2,-2}+\frac{1}{2} \sqrt{B}\left(\widetilde{b}_{n, k+1}^{2,1}+2 k \widetilde{b}_{n, k-1}^{2,1}\right), \\
{\left[\eta s+2 n \rho(s)+k \rho_{z}(s) / B\right] \widetilde{b}_{n, k}^{2,2}} \\
=-n \widetilde{b}_{n, k}^{2,1}-4(n+1) \widetilde{b}_{n-1, k}^{2,1}-\sqrt{B}\left(\widetilde{b}_{n, k}^{2,-2}+2 k \widetilde{b}_{n, k}^{2,-2}\right),
\end{gathered}
$$

$$
\begin{aligned}
{[\eta s} & \left.+2 n \rho(s)+k \rho_{z}(s) / B\right] \widetilde{b}_{n, k}^{2,-2} \\
& =-n \widetilde{b}_{n, k}^{2,-1}-4(n+1) \widetilde{b}_{n-1, k}^{2,-1}+\sqrt{B}\left(\widetilde{b}_{n, k+1}^{2,2}+2 k \widetilde{b}_{n, k-1}^{2,2}\right),
\end{aligned}
$$

Then the hierarchy of equations for $\widetilde{b}_{n, k}^{2, m}(s)$ Eqs. (B1)-(B5) can be transformed into the matrix three-term differentialrecurrence equation

$$
\begin{aligned}
& {\left[\eta s \mathbf{I}_{5 n}-\mathbf{Q}_{n}(s)\right] \widetilde{\mathbf{C}}_{n}(s)-\mathbf{Q}_{n}^{+} \widetilde{\mathbf{C}}_{n+1}(s)-\mathbf{Q}_{n}^{-} \widetilde{\mathbf{C}}_{n-1}(s)} \\
& \quad=\delta_{n, 1} \eta \mathbf{C}_{1}(0) \quad(n \geqslant 1)
\end{aligned}
$$

where the supercolumn vector $\widetilde{\mathbf{C}}_{n}(s)$ comprising of five subvectors

$$
\widetilde{\mathbf{C}}_{n}(s)=\left(\begin{array}{c}
\widetilde{\mathbf{c}}_{n-1}^{2,0}(s) \\
\widetilde{\mathbf{c}}_{n-1}^{2,1}(s) \\
\widetilde{\mathbf{c}}_{n-1}^{2,-1}(s) \\
\widetilde{\mathbf{c}}_{n-1}^{2,2}(s) \\
\widetilde{\mathbf{c}}_{n-1}^{2,-2}(s)
\end{array}\right), \quad \widetilde{\mathbf{c}}_{n}^{2, m}(s)=\left(\begin{array}{c}
\widetilde{b}_{n, 0}^{2, m}(s) \\
\widetilde{b}_{n-1,1}^{2, m}(s) \\
\vdots \\
\widetilde{b}_{0, n}^{2, m}(s)
\end{array}\right)
$$

and the matrices $\mathbf{Q}_{n}^{-}, \mathbf{Q}_{n}$, and $\mathbf{Q}_{n}^{+}$are given by

$$
\mathbf{Q}_{n}^{-}=\left(\begin{array}{ccccc}
\mathbf{0} & \mathbf{q}_{n}^{-} & \mathbf{0} & \mathbf{0} & \mathbf{0} \\
\mathbf{0} & \mathbf{0} & -\mathbf{p}_{n}^{-} & \mathbf{0} & \mathbf{0} \\
\mathbf{0} & \mathbf{p}_{n}^{-} & \mathbf{0} & \mathbf{0} & \mathbf{0} \\
\mathbf{0} & \mathbf{v}_{n}^{-} & \mathbf{0} & \mathbf{0} & -2 \mathbf{p}_{n}^{-} \\
\mathbf{0} & \mathbf{0} & \mathbf{v}_{n}^{-} & 2 \mathbf{p}_{n}^{-} & \mathbf{0}
\end{array}\right)
$$




$$
\begin{gathered}
\mathbf{Q}_{n}^{+}=\left(\begin{array}{ccccc}
\mathbf{0} & \mathbf{0} & \mathbf{0} & \mathbf{0} & \mathbf{0} \\
3 \mathbf{q}_{n}^{+} & \mathbf{0} & -\mathbf{p}_{n}^{+} & \mathbf{v}_{n}^{+} & \mathbf{0} \\
\mathbf{0} & \mathbf{p}_{n}^{+} & \mathbf{0} & \mathbf{0} & \mathbf{v}_{n}^{+} \\
\mathbf{0} & \mathbf{0} & \mathbf{0} & \mathbf{0} & -2 \mathbf{p}_{n}^{+} \\
\mathbf{0} & \mathbf{0} & \mathbf{0} & 2 \mathbf{p}_{n}^{+} & \mathbf{0}
\end{array}\right), \\
\mathbf{Q}_{n}(s)=\left(\begin{array}{ccccc}
\mathbf{q}_{n}^{0}(s) & \mathbf{I}_{n} / 2 & \mathbf{0} & \mathbf{0} & \mathbf{0} \\
6\left(\mathbf{r}_{n}-\mathbf{I}_{n}\right) & \mathbf{q}_{n}^{1}(s) & \mathbf{0} & \mathbf{I}_{n} & \mathbf{0} \\
\mathbf{0} & \mathbf{0} & \mathbf{q}_{n}^{1}(s) & \mathbf{0} & \mathbf{I}_{n} \\
\mathbf{0} & \mathbf{r}_{n} & \mathbf{0} & \mathbf{q}_{n}^{0}(s) & \mathbf{0} \\
\mathbf{0} & \mathbf{0} & \mathbf{r}_{n} & \mathbf{0} & \mathbf{q}_{n}^{0}(s)
\end{array}\right) .
\end{gathered}
$$

Here the submatrices $\mathbf{p}_{n}^{ \pm}, \mathbf{q}_{n}^{ \pm}, \mathbf{r}_{n}$, and $\mathbf{q}_{n}^{M}(s)$ are defined above and the submatrices $\mathbf{v}_{n}^{ \pm}$are

$$
\begin{aligned}
& \mathbf{v}_{n}^{-}=-4\left(\begin{array}{cccc}
n & 0 & \cdots & 0 \\
0 & n-1 & \cdots & 0 \\
\vdots & \vdots & \ddots & \vdots \\
0 & 0 & \cdots & 2 \\
0 & 0 & \cdots & 0
\end{array}\right)_{n \times(n-1)}, \\
& \mathbf{v}_{n}^{+}=\frac{1}{4}\left(\begin{array}{ccccc}
1 & 0 & \cdots & 0 & 0 \\
0 & 1 & \cdots & 0 & 0 \\
\vdots & \vdots & \ddots & \vdots & \vdots \\
0 & 0 & \cdots & 1 & 0
\end{array}\right)_{n \times(n+1)} .
\end{aligned}
$$

The exact solution, for the Laplace transform $\widetilde{\mathbf{C}}_{1}(s)$ is then given by the matrix continued fraction, viz.,

$$
\widetilde{\mathbf{C}}_{1}(s)=\eta \frac{\mathbf{I}_{5}}{\eta s \mathbf{I}_{5}-\mathbf{Q}_{1}-\mathbf{Q}_{1}^{+} \frac{\mathbf{I}_{10}}{\eta s \mathbf{I}_{10}-\mathbf{Q}_{2}-\mathbf{Q}_{2}^{+} \frac{\mathbf{I}_{15}}{\eta s \mathbf{I}_{15}-\mathbf{Q}_{3} \cdot \cdot} \mathbf{Q}_{3}^{-}} \mathbf{Q}_{2}^{-}} \mathbf{C}_{1}(0)
$$

with initial conditions

$$
\mathbf{C}_{1}(0)=\left(\begin{array}{c}
\xi^{2} / 15 \\
0 \\
0 \\
0 \\
0
\end{array}\right)
$$

and $\mathbf{C}_{n}(0)=\mathbf{0}$ for all $n \geqslant 2$.

As an example the results of numerical calculations for linear $\left(I_{z}=0\right)$ molecules based on the above matrix continued fraction solution have been compared with that of Ref. [11] presented in terms of ordinary continued fractions. The numerical calculations show that both matrix and ordinary continued fraction solutions yield the same results.
[1] E. W. Montroll and M. F. Shlesinger, in Non Equilibrium Phenomena II from Stochastics to Hydrodynamics, edited by J. L. Lebowitz and E. W. Montroll (Elsevier Science Publishers, Amsterdam, 1984).

[2] E. W. Montroll and G. H. Weiss, J. Math. Phys. 6, 167 (1965).

[3] W. T. Coffey, Yu. P. Kalmykov, and S. V. Titov, J. Chem. Phys. 116, 6422 (2002).

[4] J. R. McConnell, Rotational Brownian Motion and Dielectric Theory (Academic, New York, 1980).

[5] M. W. Evans, G. J. Evans, W. T. Coffey, and P. Grigolini, Molecular Dynamics and Theory of Broadband Spectroscopy (Wiley, New York, 1982).

[6] A. I. Burshtein and S. I. Temkin Spectroscopy of Molecular Rotation in Gases and Liquids (Cambridge University Press, Cambridge, 1994).

[7] R. Metzler, Phys. Rev. E 62, 6233 (2000).

[8] R. Metzler and J. Klafter, Adv. Chem. Phys. 116, 223 (2001).

[9] E. Barkai and R. S. Silbey, J. Phys. Chem. B 104, 3866 (2000).

[10] W. T. Coffey, Yu. P. Kalmykov, and S. V. Titov, Phys. Rev. E 65, 032102 (2002).
[11] W. T. Coffey, Yu. P. Kalmykov, and S. V. Titov, Phys. Rev. E 65, 051105 (2002).

[12] E. P. Gross, J. Chem. Phys. 23, 1415 (1955).

[13] R. A. Sack, Proc. Phys. Soc. London, Sect. B 70, 402 (1957).

[14] R. A. Sack, Proc. Phys. Soc. London, Sect. B 70, 414 (1957).

[15] A. Morita, J. Chem. Phys. 76, 3198 (1982).

[16] W. T. Coffey, Yu. P. Kalmykov, and S. V. Titov, J. Phys. A 36, 4947 (2003).

[17] R. Metzler and J. Klafter, Adv. Chem. Phys. 116, 223 (2001).

[18] S. Chapman and T. G. Cowling, The Mathematical Theory of Non-Uniform Gases, 3rd ed. (Cambridge University Press, London, 1970).

[19] J. H. Jeans, The Dynamical Theory of Gases, 4th ed. (Cambridge University Press, London, 1925).

[20] Handbook of Mathematical Functions, edited by M. Abramowitz and I. Stegun (Dover, New York, 1964).

[21] R. Carmichael, A Treatise on the Calculus of Operations (Longmans Green, London, 1855).

[22] E. C. Titchmarsh, An Introduction to the Theory of Fourier Integrals (Oxford University Press, London, 1937). 
[23] W. T. Coffey, Yu. P. Kalmykov, and J. T. Waldron, The Langevin Equation, 2nd ed. (World Scientific, Singapore, 2004).

[24] K. S. Cole and R. H. Cole, J. Chem. Phys. 9, 341 (1941).

[25] G. Williams and D. C. Watts, Trans. Faraday Soc. 66, 80 (1970).
[26] W. T. Coffey, Yu. P. Kalmykov, and S. V. Titov, J. Phys. A 35, 6789 (2002).

[27] C. J. Reid and M. W. Evans, J. Chem. Soc., Faraday Trans. 75, 1369 (1979). 